\title{
Resilient Modulus of Unbound Aggregate Base Courses from Senegal (West Africa)
}

\author{
Makhaly Ba, Meissa Fall, Fatou Samb, Déthié Sarr, Mapathé Ndiaye \\ Department of Geotechnical Engineering, University of Thiès, Thiès, Senegal \\ E-mail:makhaly.ba@univ-thies.sn \\ Received August 13, 2011; revised September 13, 2011; accepted September 20, 2011
}

\begin{abstract}
This paper presents the results of research conducted to investigate the Resilient Modulus $(\mathrm{Mr})$ of unbound aggregates used as pavement layer in Senegal (West Africa) as well as the effect of water content and density on the Resilient Modulus of the materials tested. Four different aggregates was collected from different sites within Senegal and then subjected to repeated load triaxial tests. Test results showed that the Bandia limestone is around $44 \%$ stiffer than the basalt, and $71 \%$ to $104 \%$ stiffer that the Black and the Red quartzites (GNB and GRB). The basalt is 21\% to 43\% stiffer than the GNB and the GRB. Basalt specimens compacted at Wopt- $2 \%$ were $30 \%$ stiffer than basalt specimens compacted at Wopt and $40 \%$ stiffer than those compacted at Wopt $+2 \%$. The Summary Resilient Modulus (SRM) at Wopt- 2\% is 22\% higher than SRM at Wopt and 35\% higher than SRM at Wopt+ 2\% for the GRB and the GNB. The SRM at Wopt- 2\% is 30\% higher than SRM at Wopt and 40\% higher than SRM at Wopt+ 2\%, for the Basalt. For the Bandia limestone, the SRM at Wopt- 2\% is $81 \%$ higher than SRM at Wopt and $126 \%$ higher than SRM at Wopt+ $2 \%$. Results show also that the Resilient Modulus increases around 25\% when relative density increases from $77 \%$ to $119 \%$ and the variation is more significant at high stress states than at low stress state. Results of statistical analysis and coefficients of determination $\left(\mathrm{R}^{2}\right)$ showed that the Uzan and NCHRP models are more suitable to predict the Resilient Modulus of the aggregates tested.
\end{abstract}

Keywords: Resilient Modulus, Summary Resilient Modulus, Quartzite, Basalt, Bandia Limestone, Unbound Aggregates

\section{Introduction}

Achieving a proper modulus for an unbound base course is important for pavement performance [1]. One commonly used parameter to define material stiffness is the Resilient Modulus $(\mathrm{Mr})$, which is similar to Young's modulus based on the recoverable axial strain under an imposed axial (deviator) stress. In Senegal as in a lot of developing countries, road specifications are primarily based on the material characterization but rarely on the real mechanical behavior of materials [2]. Indeed, cracking and rutting are the main modes of flexible pavement failures. These are mainly due to tensile stresses and accumulation of permanent strains over the different layers. Therefore, a rational design of flexible pavements passes necessarily by a good modeling of the mechanical behavior of these materials. Unfortunately, this mechanical behavior is poorly taken into account and, the design methods do not reflect well the rheological behavior of these materials. In the current method of pavement design in Senegal, determining the mechanical behavior of materials is usually done by a calculation in linear elasticity and the behavior is described by two constant parameters that are the Young modulus (E) and the Poisson ratio (v). However, under traffic loading, the behavior of unbound granular materials is rather nonlinear. This calls into question the inputs of these different methods, which use a linear elasticity theory to describe a nonlinear elastoplastic phenomenon. These shortcomings are led to the development of Resilient Modulus for measuring nonlinear elastic properties of unbound granular materials. This underlines the interest of the repeated load triaxial test that can characterize the behavior of materials related to the granular mixture, more representative of the 
state of the material in the pavement, but not only from characteristics of its aggregates. However, no studies on the mechanical behavior of crushed granular materials under cyclic loading has been conducted in Senegal where the interest of this work which will investigate the Resilient Modulus of granular materials to have input parameters for a mechanistic design approach in Senegal. These results will be the first obtained on unbound granular material from Senegal.

\section{Background}

The Resilient Modulus ( $\mathrm{Mr}$ ) is an elastic modulus based on the recoverable strain under repeated loads. It is defined as Equation (1) where $\sigma_{d}$ is the applied deviatoric stress $\left(\sigma_{1}-\sigma_{3}\right)$ and $\varepsilon_{r}$ is the recoverable strain.

$$
M r=\frac{\sigma_{d}}{\varepsilon_{r}}
$$

A number of factors affect the $M r$ of unbound granular materials, some of which are stress history, moisture content, density, aggregate type, gradation, percent fines [3]. A number of researchers have developed models to predict the $\mathrm{Mr}$ of granular materials [4]. However, for this research study, a Summary Resilient Modulus was calculated using the bulk stress model [5] and calculated with $\theta=208 \mathrm{kPa}$. This model can be expressed as follows:

$$
M r=k_{1}\left(\frac{\theta}{P_{a}}\right)^{k_{2}}
$$

where $\theta=\sigma_{1}+2 \sigma_{3}$ is the bulk stress; $k_{1}$ and $k_{2}$ are the material properties determined from regression analyses.

\section{Materials Characterization and Testing Procedure}

\subsection{Materials}

Resilient Modulus tests were conducted on four (04) types of crushed aggregates collected from different locations corresponding to the main sources of aggregate within Senegal: Red quartzite from Bakel (GRB), Black quartzite from Bakel (GNB), Basalt from Diack (BAS), and Limestone from Bandia (BAN). Each specimen was labeled "letter_number1_number2," where the letter represented the sample identification, number1 indicated the moisture content, and number2 indicated the dry unit weight. Grain size distributions for the materials tested are shown in Figure 1. Maximum and minimum dry unit weight and compaction characterization are presented in Table 1. Moreover, the repeated load triaxial test was used to determine the Resilient Modulus of these aggregates.

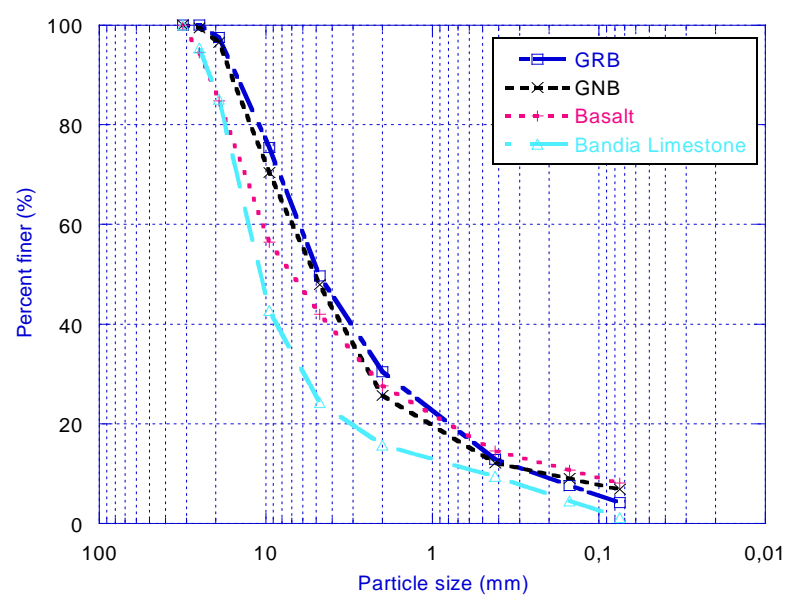

Figure 1. Particles sizes distribution for the 4 materials.

Table 1. Relative density vs. relative compaction for the four materials.

\begin{tabular}{ccccc}
\hline \multirow{2}{*}{ Materials } & \multicolumn{2}{c}{ Relative Density } & \multicolumn{2}{c}{ Compaction test } \\
\cline { 2 - 5 } & $\gamma_{\mathrm{d} \min }\left(\mathrm{kg} / \mathrm{m}^{3}\right)$ & $\gamma_{\mathrm{dmax}}\left(\mathrm{kg} / \mathrm{m}^{3}\right)$ & $\begin{array}{c}\gamma_{\mathrm{dmax}} \\
\left(\mathrm{kg} / \mathrm{m}^{3}\right)\end{array}$ & $\begin{array}{c}\text { Wopt } \\
(\%)\end{array}$ \\
\cline { 2 - 5 } GRB & 1656 & 2002 & 2140 & 5.5 \\
GNB & 1644 & 2000 & 2150 & 4.5 \\
BAS & 1890 & 2240 & 2420 & 4.2 \\
BAN & - & - & 2065 & 7.6 \\
\hline
\end{tabular}

\subsection{Resilient Modulus Test Procedure}

The cyclic loading triaxial tests were performed using a MTS closed-loop servo-electrohydraulic testing system which is capable of applying repeated load in haversine waveform with a wide range of load duration. The axial deformations were measured by LVDTs mounted inside the triaxial cell. The specimens were submitted to cyclic loading triaxial tests according to the NCHRP 1-28A [6] test protocol, which was used to establish the 30 loading sequences. The loading involves conditioning, which attempts to establish steady-state or resilient behavior, through the application of 1000 cycles of $207 \mathrm{kPa}$ deviator stress at $103.5 \mathrm{kPa}$ confining pressure. The cycles are then repeated 100 times for 30 loading sequences with different combinations of deviator stress and confining pressure. The $\mathrm{Mr}$ is calculated as the mean of the last five cycles of each sequence from the recoverable axial strain and cyclic axial stress.

\section{Test Results and Analyses}

$M r$ versus confining pressure plots for the four different 
materials compacted at their optimum moisture contents and at $98 \%$ of the maximum dry unit weight for the GRB, GNB and the basalt, 95\% of the maximum dry unit weight for the limestone are shown in Figure 2. The spread in the data at a constant confining pressure represents the $M r$ at various deviator stresses. The curve fit is based on power dependence on confinement. Typical of granular materials, the $\mathrm{Mr}$ increased consistently with increase of confining pressure. Bandia limestone is around $44 \%$ stiffer than the basalt, and $71 \%$ to $104 \%$ stiffer that the GNB and the GRB. The basalt is $21 \%$ to $43 \%$ stiffer than the GNB and the GRB. The difference of Resilient Modulus between the GRB and the GNB doesn't exceed $10 \%$.

A summary of the $M r$ results is presented in Figure 3, for Basalt sample, compacted at three moisture contents (Wopt- 2\%, Wopt and Wopt+2\%). The results show that specimens compacted at Wopt- $2 \%$ exhibited the highest $\mathrm{Mr}$, followed by the specimen compacted at Wopt, and specimen compacted at Wopt $+2 \%$ exhibited the lowest $M r$. Specimens compacted at Wopt- 2 was 30\% stiffer than those compacted at Wopt and 40\% stiffer than those compacted at Wopt $+2 \%$.

Figure 4 shows the variation of the Summary Resilient Moduli (SRM) with compaction water content for the four materials tested. The SRM at Wopt- $2 \%$ is $22 \%$ higher than SRM at Wopt and 35\% higher than SRM at Wopt $+2 \%$ for the GRB and the GNB. The SRM at Wopt- 2\% is 30\% higher than SRM at Wopt and 40\% higher than SRM at Wopt+ 2\%, for the Basalt. The SRM at Wopt- $2 \%$ is $81 \%$ higher than SRM at Wopt and $126 \%$ higher than SRM at Wopt+ $2 \%$ for the Bandia limestone. Then, The Bandia limestone is much more sensitive to water content than the GRB, GNB and Basalt.

Variation of Resilient Modulus values as a function of dry density was also determined for the GRB and the GNB. Resilient Modulus values obtained from the bulk model are shown in Figures $\mathbf{5}$ and $\mathbf{6}$ for three selected stress states representing lower, intermediate and higher states of stress. At the intermediate stress state, Resilient Modulus of the GRB increases from $147 \mathrm{MPa}$ to 186 $\mathrm{MPa}$, and from $181 \mathrm{MPa}$ to $222 \mathrm{MPa}$ for the GNB for relative density ranging from $83 \%$ to $119 \%$ and $77 \%$ to $119 \%$, respectively. At higher stress level, Resilient Modulus of the GRB increases from $235 \mathrm{MPa}$ to 297 MPa, while the Resilient Modulus of the GNB increases from $287 \mathrm{MPa}$ to $331 \mathrm{MPa}$ for the GNB and for relative density ranging from $83 \%$ to $119 \%$ and $77 \%$ to $119 \%$, respectively. These results show that the Resilient Modulus increases around 25\% for relative density ranging from $77 \%$ to $119 \%$ and the variation is more significant at high stress state than at low stress state.

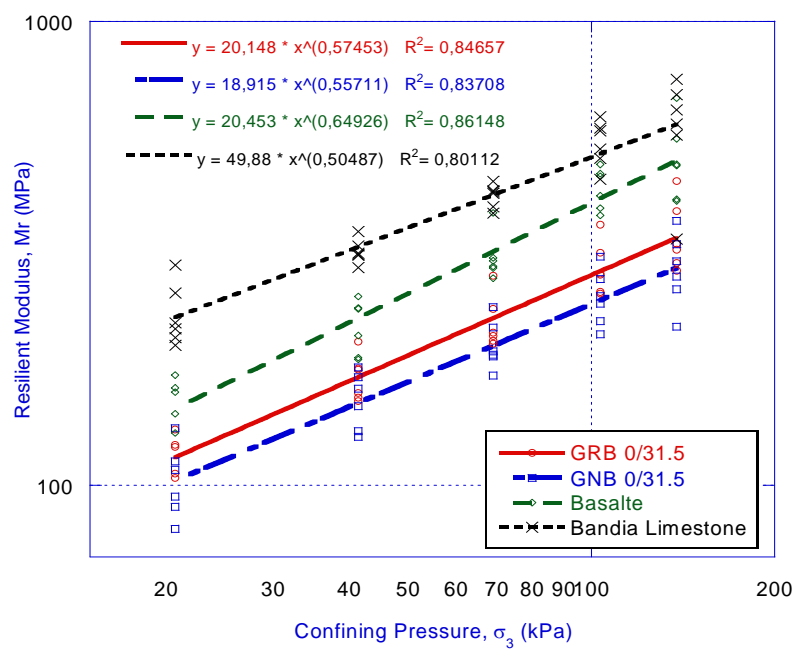

Figure 2. Comparison between $M r$ of different materials tested at their optimum water content.

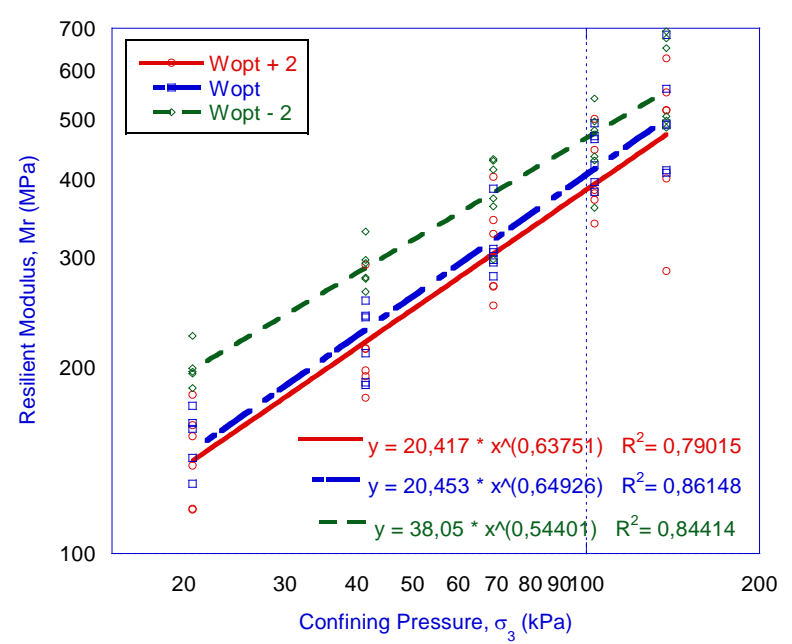

Figure 3. Effect of water content on the Resilient Modulus for the Basalt.

\section{Regression Analysis of the Resilient Modulus Test Results}

There are several models that were developed for the estimation of Resilient Modulus of unbound granular materials [5] and [7]. The Seed model is specified as bulk model (equation 1) and the Uzan model is known as universal model (Equation 2)

$$
M r=k_{1} P_{a}\left(\frac{\theta}{P_{a}}\right)^{k_{2}}\left(\frac{\sigma_{d}}{P_{a}}\right)^{k_{3}}
$$

where $M r$ is the Resilient Modulus, $\sigma_{d}$ is the deviator stress, $\theta$ is the bulk stress, $P_{a}$ is the atmospheric pressure (used to normalize $M r$ units), and $k_{1}, k_{2}$ and $k_{3}$ are material constants. 


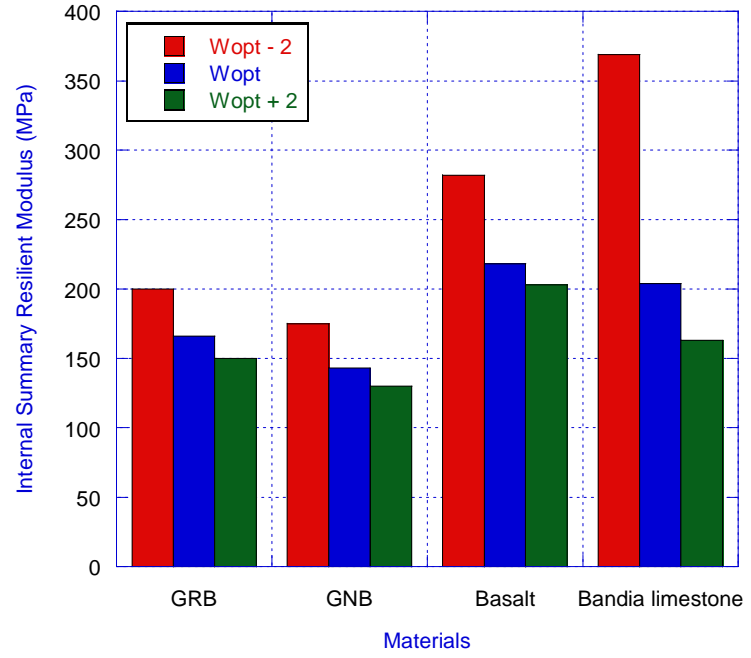

Figure 4. Variation of the summary resilient modulus with water content for GRB, GNB, Basalt and Bandia Limestone.



Figure 5. Effect of relative density on the resilient Modulus calculated from the power model (GRB).

A new "harmonized" Resilient Modulus test protocol was developed through the NCHRP project 1-28A [6]. This model called either NCHRP model or MEPDG model is implemented in the new "Mechanistic-Empirical Pavement Design Guide" (MEPDG). The new protocol uses the universal nonlinear model that is applicable for unbound base or subbase materials Equation (3):

$$
M r=k_{1} P_{a}\left(\frac{\theta}{P_{a}}\right)^{k_{2}}\left(\frac{\tau_{o c t}}{P_{a}}+1\right)^{k_{3}}
$$

where $M r$ is the Resilient Modulus, $\sigma_{d}$ is the deviator stress, $\theta$ is the bulk stress $\left(=\sigma_{1}+\sigma_{2}+\sigma_{3}\right), \tau_{o c t}$ is the octahedral shear stress, $P_{a}$ is the atmospheric pressure (used to normalize $M r$ units), and $k_{1}, k_{2}$ and $k_{3}$ are material constants.



Figure 6. Effect of relative density on the resilient modulus calculated from the power model (GNB).

In this paper, these three models were used to characterize the Resilient Modulus of the investigated aggregate base courses. Regression analysis was conducted to evaluate the material constants $k_{1}, k_{2}$ and $k_{3}$. The GRB, GNB and the Basalt are compacted at Wopt- $2 \%$ and $98 \%$ of the maximum dry unit weight, while the Bandia limestone is compacted à Wopt- $2 \%$ and $95 \%$ of the maximum dry unit weight. Results of the statistical analysis are summarized in Table 2. The coefficient of determination $\left(\mathrm{R}^{2}\right)$ was calculated for each sample tested to provide information about the regression analysis.

Figures 7-10 represent the variation of measured Resilient Moduli with the predicted Resilient Moduli from Seed, Uzan and NCHRP models for BAN_5.80_1956, GNB0/31.5_2.08_1921, BAS_3.90_2417 and GRB 0/31.5_00_2042 samples. These results show that the Uzan and NCHRP models are more suitable to predict the Resilient Modulus of the aggregates tested.

\section{Conclusions}

Repeated load triaxial test was conducted on four different aggregates collected from different sites within Senegal (West Africa) in order to determine the Resilient Moduli of these aggregates. Aggregate specimens were subjected to Resilient Modulus test in accordance with the NCHRP project 1-28A [6]. Tests results showed that the Bandia limestone exhibit the higher Resilient Moduli, followed by the Basalt and the GNB and GRB. Test results showed also that the Resilient Modulus is significantly affected by the water content for the limestone and less affected by water content for the GNB, the GRB and the basalt tested. Specimens compacted with different density showed that the Resilient Modulus increases 
Table 2. Results of the statistical analysis from Seed, Uzan and NCHRP models.

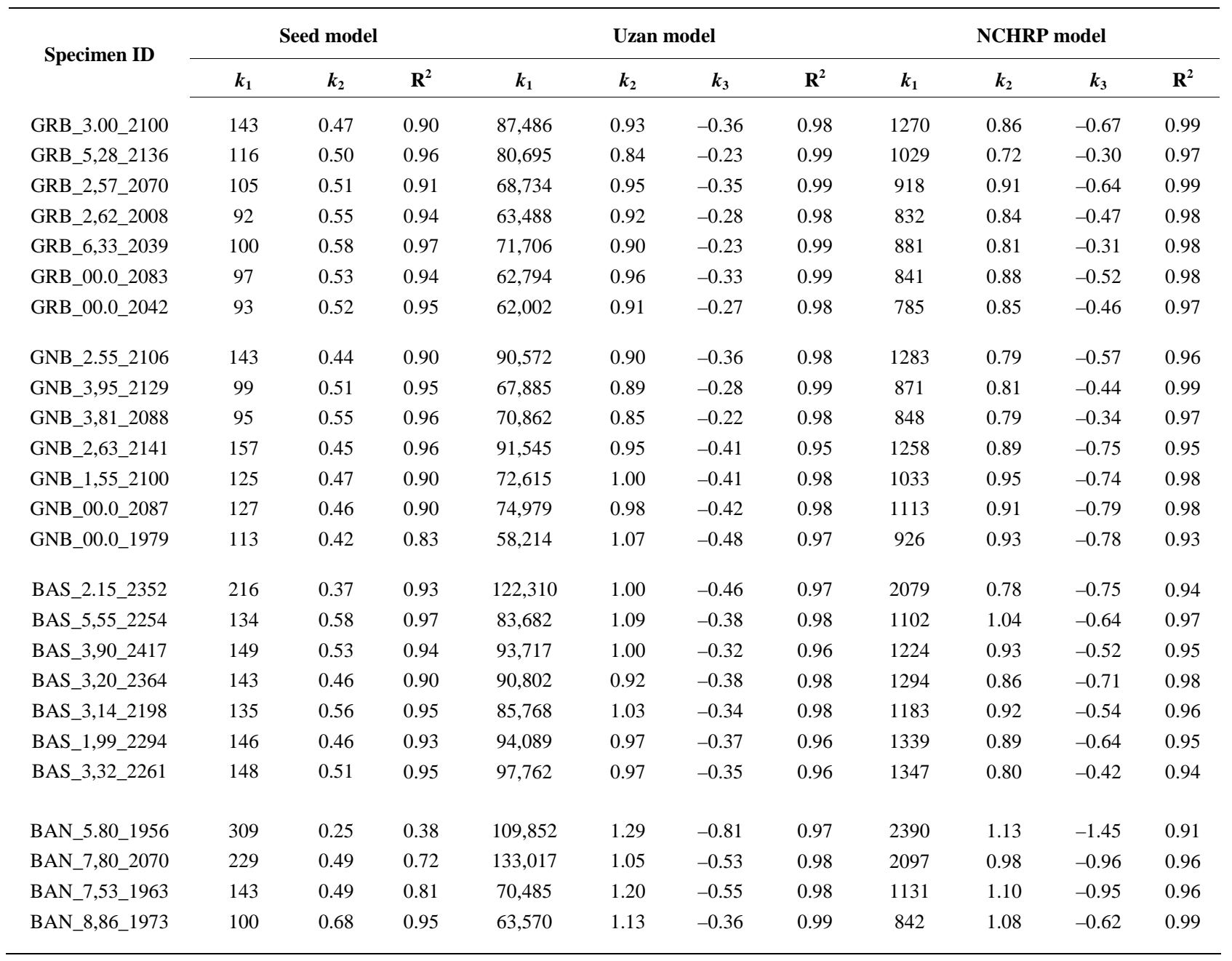

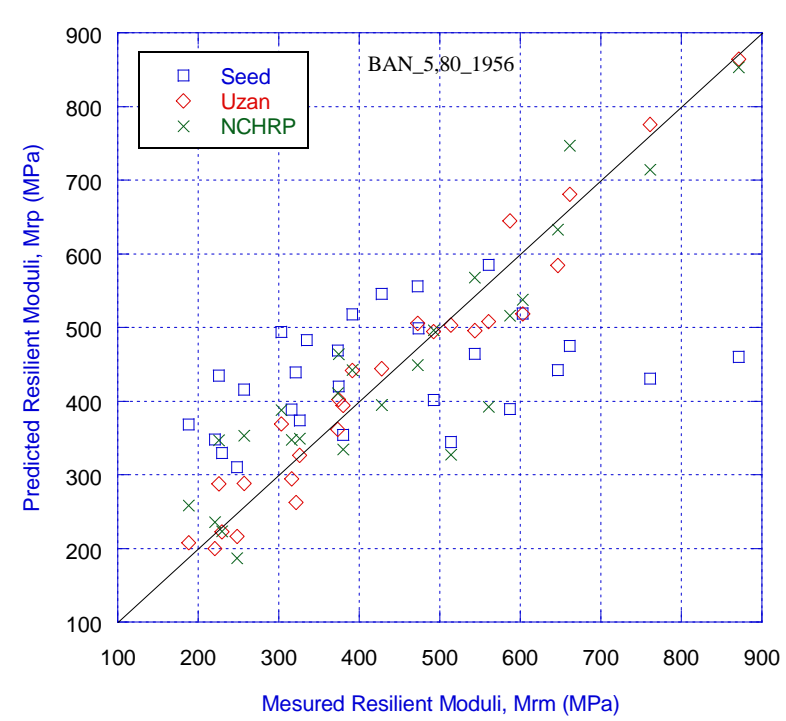

Figure 7. Measured vs. predicted Resilient Moduli from Seed, Uzan and NCHRP models (BAN_5.80_1956 sample).



Figure 8. Measured vs. predicted Resilient Moduli from Seed, Uzan and NCHRP models (GNB 0/31.5_2.08_1921 sample). 


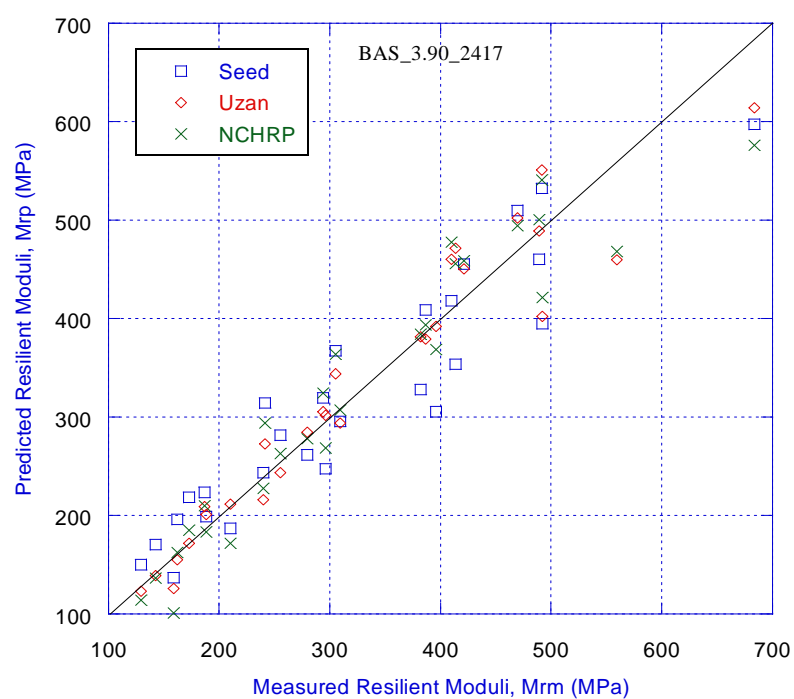

Figure 9. Measured vs. predicted Resilient Moduli from Seed, Uzan and NCHRP models (BAS_3.90_2417 sample).

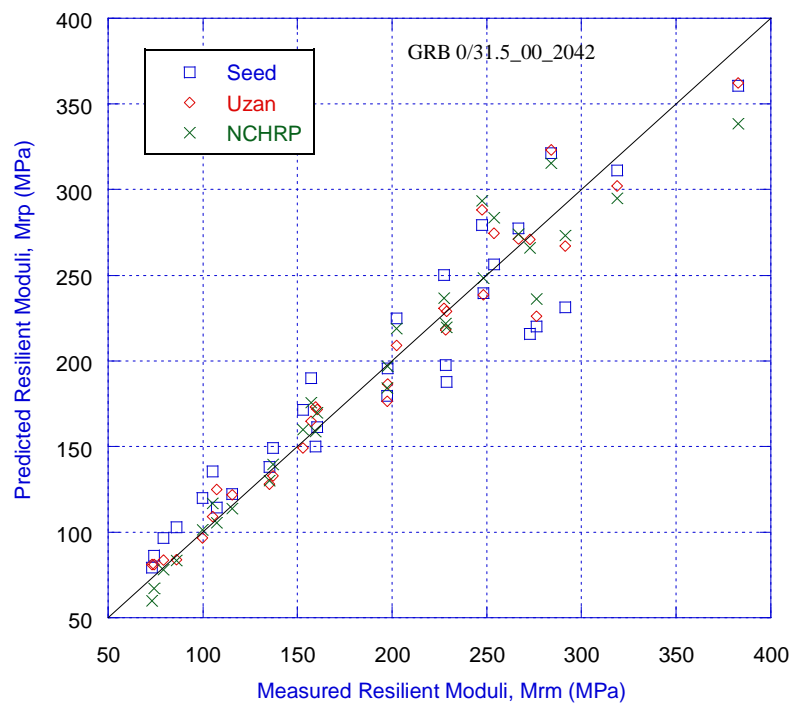

Figure 10. Measured vs. predicted Resilient Moduli from Seed, Uzan and NCHRP models (GRB 0/31.5_00_2042 sample). around 25\% for relative density ranging from $77 \%$ to $119 \%$ and the variation was more significant at high stress states than at low stress states.

\section{Acknowledgements}

The authors would like to acknowledge the Geo-Engneering research group of the University of Wisconsin-Madison for their guidance and valuable input in this research project; and the "Entreprise Mapathé NDIOUCK” for supporting the high price shipping of aggregates from Senegal to United States of America.

\section{References}

[1] E. J. Yoder and M. W. Witczak, "Principles of Pavement Design,” $2^{\text {nd }}$ Edition, Wiley, New York, 1975.

[2] M. Fall, A. Sawangsuriya, C. H. Benson, T. B. Edil and P. J. Bosscher, "On the Investigations of Resilient Modulus of Residual Tropical Gravel Lateritic Soils from Senegal (West Africa)," Geotechnical and Geological Engineering Journal, Vol. 26, No. 1, 2008, pp. 13-35.

[3] R. G. Hicks and C. L. Monismith, "Factors Influencing the Resilient Properties of Granular Materials," Ph.D. Thesis, University of California, Berkeley, 1970.

[4] F. Lekarp, U. Isacsson and A. Dawson, "State of the Art. I: Resilient Response of Unbound Aggregates," Journal of Transportation Engineering, Vol. 126, No. 1, 2000, pp. 66-75.

[5] H. B. Seed, F. G. Mitry, C. L. Monismith and C. K. Chan, "Prediction of Flexible Pavement Deflections from Laboratory Repeated Load Tests,” National Academy of Sciences-National Academy of Engineering, Washington, 1967.

[6] NCHRP, "Laboratory Determination of Resilient Modulus for flexible Pavement Design,” National Cooperative Highway Research Program (NCHRP), Transportation Research Board of National Academies, Washington, 2004.

[7] J. Uzan, “Characterization of Granular Material,” Transportation Research Board, Washington, 1985, pp. 52-59. 